\title{
¿Cómo innovan las fintech peruanas? \\ Una aproximación a partir de un estudio de casos múltiple
}

\section{How do Peruvian fintech innovate? \\ An approach from a multiple case study.}

\author{
Gianella Judith Palomino Barchi \\ BBVA Perú \\ gianella.palomino@pucp.pe \\ Katherinne Vanessa Velásquez Bondia \\ Hunt Oil Exploration and Production Company of Perú \\ vanessa.velasquez@pucp.pe \\ Katharine Cledia Marcos Quispe \\ Pontificia Universidad Católica del Perú \\ katharine.marcos@pucp.pe \\ Jean Pierre Seclen Luna \\ Pontificia Universidad Católica del Perú \\ iseclen@pucp.pe
}


Las fintech son un tipo de startup con un modelo de negocio disruptivo que brinda servicios financieros digitales. Una de sus principales ventajas competitivas es que innovan continuamente, por lo cual la gestión de la innovación resulta relevante. En Perú, si bien el ecosistema fintech está creciendo, no hay estudios empíricos que traten sobre innovación en fintech. Este artículo aporta al conocimiento sobre las fintech, ya que analiza la gestión de sus procesos de innovación mediante la identificación de habilitadores y prácticas claves propios del proceso. Para ello, se realizó un estudio de casos múltiple de seis fintech ganadoras del concurso Startup Perú. Los hallazgos demuestran que, por la naturaleza iterativa y ágil de las fintech, algunas no cuentan con un proceso de innovación sistematizado; sin embargo, existen habilitadores y prácticas claves de gestión del proceso de innovación que les permiten lanzar nuevos servicios al mercado de manera exitosa.

Palabras clave: fintech, innovación, gestión de la innovación, proceso de innovación

Fintech is a type of startup with a disruptive business model that provides digital financial services. One of its main competitive advantages is that they continually innovate, which is why the management of the resulting innovation relevant to them. In Peru, although the fintech ecosystem is growing, there are no empirical studies dealing with innovation in fintech. This article contributes to the knowledge of fintech and analyzes the management of its innovation processes by identifying key enablers and practices of the process. For this, a multiple case study of six fintech winners of the Startup Peru contest was carried out. The findings we have, due to the iterative and agile nature of fintech startups, some do not have a process of systematized innovation; however, there are key enablers and practices for managing the innovation process that allow them to successfully launch new services to the market. 


\section{Introducción}

En la actualidad, el sistema financiero peruano se ha encontrado con un nuevo actor, las financial technologies — también llamadas fintech—, un tipo de startup que utiliza la tecnología para ofrecer productos y servicios financieros innovadores (Rojas, 2017). Una de sus principales ventajas competitivas es el uso de la innovación tecnológica como modelo de negocio (Rojas, 2016); por ello, la gestión de la innovación resulta un campo de investigación interesante en este tipo de startup.

Perú, si bien registró un crecimiento muy alto en el número de fintech, pasando de 16 fintech en el 2017 a 57 en el 2018, resulta ser el segundo país del sector en Latinoamérica con la tasa de mortalidad más alta, 19\%, cifra que indica la alta competencia que existe en el sector financiero peruano a la hora de incorporar soluciones innovadoras y tecnológicas al mercado (BID, BID Invest \& Finnovista, 2018). Frente a este contexto competitivo, la forma en la que las fintech gestionan sus procesos de innovación resulta ser un factor clave para mantener su competitividad y continuidad en el mercado (Dodgson, Gann \& Phillips, 2014). En este sentido, es importante que cuenten con un proceso de innovación sistematizado y estructurado, ya que el éxito de una empresa consiste en que esta sea capaz de gestionar este proceso de manera consciente (Seclen Luna, 2019).

Luego de una revisión de la literatura existente relacionada a la gestión del proceso de innovación, se identificó que esta no ha sido estudiada a profundidad en las fintech. Así, aunque existen estudios empíricos relacionados a la gestión del proceso de innovación en diferentes tipos de empresas, son pocos los que tienen como sujeto de estudio a empresas del sector financiero (Khraisha \& Arthur, 2018). Al respecto, un reciente estudio que trata la innovación en fintech en Finlandia concluye que se necesita una comprensión más profunda de fintech en lo que respecta a la innovación de servicios financieros (Riikkinen, Saraniemi \& Still, 2019).

En este estudio se considera que no contar con un proceso de innovación de servicios formalizado y estructurado en los emprendimientos fintech resultaría en una reducción de su competitividad en el mercado (Froehle, Roth, Chase \& Voss, 2000; Dodgson et al., 2014; Seclen Luna, 2019). Además, se asume que las fintech beneficiarias del concurso Startup Perú son conscientes de la importancia de innovar continuamente y, por ende, son propensas a sistematizar sus procesos de innovación. En este sentido, el objetivo de esta investigación es analizar la gestión del proceso de innovación de fintech peruanas mediante un estudio de casos múltiples de seis fintech ganadoras del concurso StartUp Perú. En particular, teniendo como base el modelo de ciclo de proceso de desarrollo de nuevos servicios (NSD, por sus siglas en inglés) de Johnson, Menor, Roth y Chase (2000), se identifican y caracterizan los habilitadores y las prácticas claves de gestión del proceso de innovación de estas fintech.

La estructura de este artículo es la siguiente: primero, se establece un marco teórico sobre la innovación y la importancia de la gestión del proceso de innovación en las fintech. Luego, se realiza el estudio empírico y se presentan los resultados. Después de ello, se interpretan y discuten los hallazgos, se concluye y se mencionan las limitaciones del estudio. 


\section{Marco teórico}

\subsection{Innovación y fintech}

La innovación es un proceso complejo que muchos académicos han desarrollado desde diversos enfoques buscando definir su naturaleza (Rosenberg, 1982; Nelson \& Winter, 1982; Tidd, Bessant \& Pavitt, 2005). Si bien la innovación tiene diversas definiciones, en esta investigación se considera que es un proceso mediante el cual una empresa se encarga de mejorar o crear nuevos productos, servicios, procesos, formas de comercializar o de organizarse con el objetivo de generar valor para la empresa (Seclen Luna, 2016). Por otro lado, de las diferentes formas de clasificar a la innovación, la más difundida y aceptada es la del Manual de Oslo, el cual indica que, según su naturaleza, esta puede ser de cuatro tipos: producto o servicio, proceso, organizacional y mercadotecnia (OCDE \& Eurostat, 2018). Al respecto, Seclen Luna y Barrutia-Güenega (2019) indican que estos tipos de innovación pueden complementarse entre sí, lo cual genera que la empresa sea competitiva.

Centrándonos en la innovación de servicios, el Manual de Oslo indica que se da al introducir un servicio nuevo o al añadir mejoras significativas en los procesos de entrega al cliente (OCDE \& Eurostat, 2018); además, se considera que se puede complementar con otros tipos de innovación. El sector financiero es considerado como un sector terciario donde las industrias abarcan la transformación de bienes materiales, personas o información (Freeman, 1991; Miles, 2006). Adicionalmente, la industria de los servicios financieros es vista como el sector que está a la vanguardia en el uso de tecnologías de la información (Barras, 1990). En años recientes, la tecnología financiera o fintech es vista como una de las tecnologías que podría revolucionar la industria de servicios financieros (Wonglimpiyarat, 2017). El término fintech abarca propuestas que posibilitan servicios y soluciones mediante el uso de tecnologías integradas de información. Además, las fintech ofrecen un nuevo panorama en la era digital de la industria financiera y proporcionan una plataforma para bancos y organizaciones no financieras para facilitar las transferencias entre redes y los servicios de pago (Thompson, 2017; Shim \& Shin, 2017).

Ahora bien, en esta investigación se utiliza a la definición propuesta por Khraisha y Arthur (2018), quienes entienden la innovación financiera como el proceso llevado a cabo por cualquier institución que implica la creación, promoción y adopción de productos, de plataformas y procesos nuevos o habilitadores de tecnologías que introducen nuevas formas o cambios en la forma en que se lleva a cabo la actividad financiera (Khraisha \& Arthur, 2018, p. 4). Por tanto, bajo esta perspectiva, las fintech realizan innovación financiera, ya que usan la tecnología digital y sus herramientas asociadas para prestar servicios de intermediación financiera de una forma innovadora y bajo nuevos modelos de negocio (Rojas, 2016). Además, es importante clarificar que, más allá de brindar servicios financieros más seguros y sencillos (Khraisha \& Arthur, 2018), las fintech se diferencian de las empresas de banca tradicional por su enfoque en el diseño de la tecnología, en la entrega digital del servicio y en el modelo de negocio disruptivo que poseen (Rojas, 2016; Varga, 2017).

De acuerdo con la clasificación de innovación del Manual de Oslo antes mencionado, Nicoletti (2017) ha desarrollado una clasificación de innovación en las fintech. De esta forma, afirma que las innovaciones en una fintech pueden ser de producto 
(desarrollo de nuevos servicios financieros digitales con la ayuda de dispositivos móviles), de proceso (nuevos métodos de creación, distribución o soporte del servicio financiero móvil), organizacional (nuevos métodos o prácticas organizativas para crear mayor contacto con los clientes, incluye la innovación en mercadotecnia) y de modelo de negocio (creación de un modelo de innovación holístico que abarca varias innovaciones, enfocado en crear, capturar y entregar valor con nuevos servicios que brinden experiencias digitales). A partir de lo expuesto, Nicoletti (2017) propone abordar el modelo de innovación de manera integral, considerando al menos cuatro variables: estructura organizativa, procesos de innovación, tecnología y personas.

\subsection{Proceso de innovación en los servicios}

La literatura sobre innovación muestra evidencia empírica de que la gestión de la innovación genera éxito competitivo y continuidad en las empresas (Dodgson et al., 2014). Sin embargo, la gestión de la innovación es un proceso complejo en el que la integración de los actores y factores internos y externos no resulta automática (Fundación COTEC, 1998). Por ello, este proceso debe ser sistematizado y coordinado con distintas áreas o departamentos de la organización (Seclen Luna, 2019; Seclen Luna \& Barrutia-Güenaga, 2019; Tidd et al., 2005).

Enfocándonos en los procesos de innovación, estos se caracterizan por ser desestructurados, inciertos, complejos y por estar conformados por un conjunto de actividades interrelacionadas, más que secuenciales (Seclen Luna \& Barrutia-Güenaga, 2019). Para su comprensión, diversos autores han desarrollado sus propios modelos de proceso de innovación en servicios, destacando el modelo general del proceso de innovación de Sundbo y Gallouj (1998); el modelo dimensional de Bilderbeek, Den Hertog, Marklund y Miles (1998); el modelo del ciclo del proceso de innovación NSD de Johnson et al. (2000); el modelo simplificado de Tidd \& Bessant (2009); y el modelo del diseño y desarrollo de los servicios de Tether (2013). Al respecto, Seclen Luna y Barrutia-Güenaga (2019) mencionan que cada organización debe tener su propio modelo de proceso de innovación en función de sus recursos, capacidades y objetivos estratégicos, lo cual no implica que todas sus fases tengan que llevarse a cabo para cada innovación.

En esta investigación tomamos como referencia el modelo de Johnson et al. (2000) o ciclo del proceso del NSD, ya que permite identificar, clasificar y explicar las principales prácticas y los habilitadores de gestión del proceso de innovación en las empresas de servicios. El modelo consta de cuatro fases (figura 1) y se caracteriza por contar con una estructura adaptable al diseño particular de servicios, poseer factores habilitadores que lo dinamicen y ser de carácter iterativo (Johnson et al., 2000). A continuación, describiremos brevemente el modelo en cuestión. 


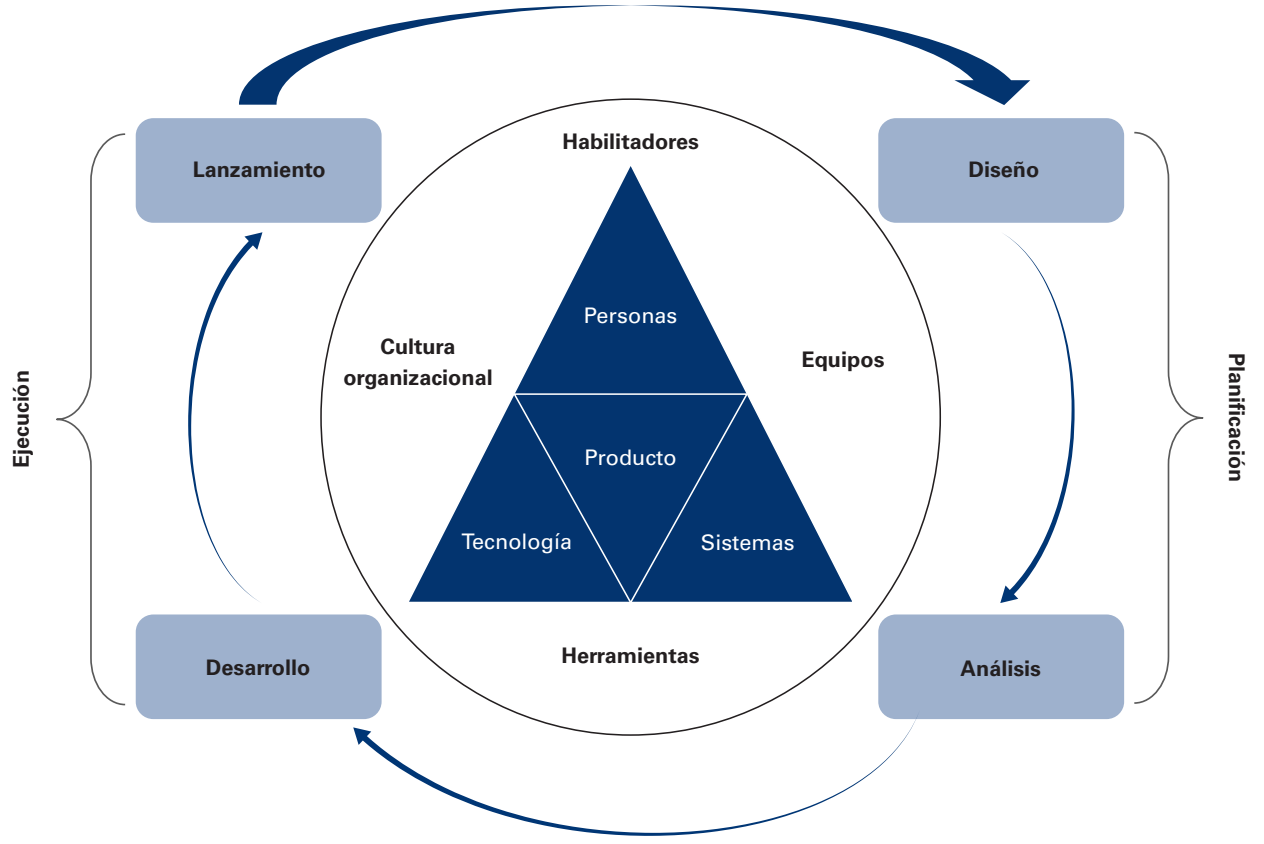

Fuente: adaptado de Johnson et al. (2000).

\subsubsection{Habilitadores del proceso de innovación}

Johnson et al. (2000) afirman que el proceso de innovación del NSD se ve impactado por «habilitadores», los cuales actúan como lubricantes que facilitan el flujo del ciclo de este proceso y se clasifican en: equipo, cultura organizacional y herramientas. En la variable equipo se identifica que la experiencia previa del equipo fundador influye en el desarrollo de innovaciones en las empresas pequeñas (Benito, Platero \& Rodríguez, 2012; Fernández, 2012; Morales, Ortíz Riaga \& Arias Cante, 2012). De igual forma, existen individuos clave que facilitan el proceso de innovación (Tidd \& Bessant, 2009).

En cuanto a la cultura organizacional, normalmente se suele emplear un concepto amplio que se refiere a los valores, normas y creencias que son duraderos en la organización (Tidd \& Bessant, 2009); así, en la presente investigación, esta se observa en base a sus aspectos formales o visibles. En cuanto a los aspectos formales o visibles, los habilitadores son la inversión en el equipo, la estructura apropiada, la visión compartida y voluntad de innovar, y el enfoque externo. Respecto a los aspectos informales, se puede mencionar el clima creativo (Tidd \& Bessant, 2009).

Finalmente, las herramientas de gestión son sistemas y/o métodos que apoyan o mejoran los procesos del NSD (Brady et al., 1997) y fortalecen de manera sistemática el conjunto de conocimientos y prácticas de la gestión de la innovación (Mogee, 1993, citado en Brady et al., 1997).

\subsubsection{El proceso de innovación en los servicios: principales prácticas}


El proceso de innovación consta de cuatro fases: diseño, análisis, desarrollo y lanzamiento (Johnson et al., 2000). La primera fase está conformada por prácticas de generación de ideas, desarrollo del concepto y testeo, y la formulación de objetivos y/o estrategias del nuevo servicio. Respecto a la generación de ideas, la empresa puede desarrollar estrategias de generación de ideas innovadoras a partir del uso de fuentes internas y externas a la empresa (Tidd \& Bessant, 2009). Para el desarrollo del concepto y testeo del nuevo servicio, Froehle y Roth (2009) recomiendan las siguientes prácticas: el aporte del cliente (que consiste en recopilar toda la información posible del cliente previamente al diseño del nuevo servicio), el enfoque en el cliente (se enfoca en satisfacer las necesidades de los clientes), la retroalimentación del cliente (busca obtener feedback de los clientes sobre el nuevo concepto del servicio) y la retroalimentación de aliados (es una práctica que consiste en buscar retroalimentación de socios y/o proveedores sobre el nuevo concepto del servicio). Por último, sobre la formulación de objetivos y/o estrategias del nuevo servicio, la práctica de definición estratégica consiste en definir y comunicar los objetivos estratégicos para el nuevo concepto del servicio (Froehle \& Roth, 2009).

En cuanto a la segunda fase del análisis, se busca evaluar críticamente el potencial financiero, estratégico y de rendimiento de mercado del nuevo servicio. En esta fase se identificaron dos grupos de prácticas: análisis de negocio y la validación del proyecto (Johnson et al., 2000). Respecto al primer grupo de prácticas, este comprende el análisis financiero (establece si existe viabilidad económica), la investigación del mercado (que consiste en realizar un análisis del mercado a profundidad para ayudar a definir el nuevo concepto del servicio antes del diseño formal), el análisis de los competidores (que se enfoca en considerar las estrategias y servicios de la competencia al evaluar si el nuevo concepto de servicio es viable) y el análisis técnico (que consiste en investigar si la empresa posee la capacidad, el tiempo y los recursos para el desarrollo). Finalmente, la validación del proyecto se da en la empresa cuando los representantes, luego de realizados los análisis, aprueban y autorizan formalmente la nueva innovación (Cowell, 1988; Froehle \& Roth, 2009).

En la tercera fase, desarrollo de la innovación, el principal propósito es traducir el concepto inicial desarrollado previamente en un servicio materializable, viable y comercializable (Cowell, 1988; Froehle \& Roth, 2009). Para ello, se requiere realizar varias actividades, tales como el diseño y prueba de los elementos tangibles del servicio y del sistema de prestación, el reclutamiento y capacitación del personal, la construcción de las instalaciones y el establecimiento de nuevos sistemas de comunicación, entre otras actividades. De esta forma, la empresa realiza una mayor inversión (Cowell, 1988). En síntesis, esta fase está compuesta por tres grupos de prácticas: diseño del desarrollo, prototipado, testeo y piloto (Johnson et al., 2000). Respecto al diseño del desarrollo, se identificó que la elaboración del diagrama de flujo de procesos (como el service blueprint) ayuda a establecer los flujos de información, las personas involucradas y los subprocesos del servicio (Froehle \& Roth, 2009). Por su parte, el prototipado consiste en el uso de herramientas de prototipo que permiten poner a prueba el sistema y los procesos del nuevo servicio (Froehle \& Roth, 2009). En cuanto a las prácticas de testeo y piloto, son las pruebas de prelanzamiento, que consisten en actividades de testeo que prueban y/o depuran parte del nuevo servicio (Froehle \& Roth, 2009); y la confirmación del mercado, que consiste en 
realizar pruebas de mercado a usuarios finales del servicio, lo que corrobora el desarrollo idóneo del mismo servicio (Froehle \& Roth, 2009).

Finalmente, la fase que culmina el proceso de innovación es el lanzamiento, cuyos grupos de prácticas son los siguientes: lanzamiento full escala y evaluación postlanzamiento (Johnson et al., 2000). Las prácticas de lanzamiento full escala son dos: el lanzamiento formalizado, el cual consiste en desarrollar e implementar un programa detallado de lanzamiento del nuevo servicio; y la promoción formalizada, que consiste en implementar un programa promocional detallado para el servicio recién introducido al mercado, para lo cual el desarrollo de publicidad es un factor relevante. Por otro lado, la práctica de evaluación postlanzamiento consiste en revisar cómo se realizó la introducción del nuevo servicio al mercado mediante recopilación del feedback de clientes a través de encuestas de satisfacción (Froehle \& Roth, 2009).

\section{Metodología}

Esta investigación pretende aproximarse al fenómeno de las fintech ganadoras del concurso StartUp Perú con el propósito de conocer y describir la gestión de sus procesos de innovación. A continuación, detallamos la metodología empleada en la investigación.

\subsection{Estrategia de investigación}

El presente artículo pretende obtener una visión más profunda de la gestión del proceso de innovación de servicios, por lo que la adopción de una estrategia de investigación cualitativa es adecuada para este estudio (Saunders, Lewis \& Thornhill, 2009). De esta forma, se emplea un estudio exploratorio de caso múltiple de tipo holístico ya que, por un lado, se analizan seis fintech; y, por otro lado, los casos son estudiados como un todo organizacional (Yin, 2003). Además, consideramos relevante esta estrategia porque es apropiado para temas poco estudiados o desarrollados, pues permite estudiar a la empresa en su estado natural con el fin de generar teorías y comprender la complejidad de los procesos que posee (Castro, 2010).

\subsection{Selección de los casos}

Perú es uno de los países que ha tomado importancia en el sector fintech puesto que, en comparación con los demás países de la región, es el segundo país que más ha crecido en ese rubro, pasando de contar con 16 emprendimientos fintech en el 2017 a presentar 57 fintech en el 2018, lo que representa un crecimiento de 256\% (BID et al., 2018). Este aumento del número de emprendimientos se da como resultado del continuo crecimiento y desarrollo del ecosistema fintech, el cual se encuentra conformado por una serie de actores del ámbito público y privado.

En particular, el papel de diversos actores del sector público ha sido clave, ya que han promovido la inclusión financiera. Una de las iniciativas de mayor calado es el concurso StartUp Perú, que es una iniciativa del Programa Nacional de Innovación para la Competitividad y Productividad (Innóvate Perú), que tiene como objetivo promover el 
surgimiento y la consolidación de nuevas empresas con alto potencial de crecimiento e innovación mediante un conjunto de concursos y líneas de financiamiento (Ministerio de la Producción, 2019). Desde su nacimiento en el 2012 a la fecha, el concurso StartUp Perú cuenta con "seis generaciones", en las cuales han participado diversas fintech y, como resultado, han sido 27 las beneficiadas con un total de S/ 2770000 de capital semilla (Javier Salinas, comunicación personal, 22 de julio, 2019).

En este contexto, se utilizó un enfoque de muestreo teórico para la selección de casos, de acuerdo con el método de investigación cualitativa (Eisenhardt \& Graebner, 2007). Los casos fueron seleccionados por conveniencia en base a los siguientes criterios: ser una fintech peruana, encontrarse en una etapa de desarrollo o expansión a la fecha de la investigación y realizar innovaciones de servicios. La variedad de casos en el sector evaluado proporcionó un gran conjunto de datos (ver tabla 1) que, en última instancia, aumentó la validez y precisión de esta investigación cualitativa (Jack \& Raturi, 2006; Saunders et al., 2009).

\section{Tabla 1. Casos de estudio}

\begin{tabular}{l|l|l} 
Nombre de fintech & Tipo de fintech & Generación \\
\hline Comparabien & Gestión de finanzas personales & $2 \mathrm{G}$ \\
Innova Factoring & Financiamiento participativo & $4 \mathrm{G}$ \\
Solven Funding & Marketplace de préstamos & $5 \mathrm{G}$ y $6 \mathrm{G}$ \\
Latin FinTech & Préstamos & $6 \mathrm{G}$ \\
Kambista & Cambio de divisas & $5 \mathrm{G}$ y $6 \mathrm{G}$ \\
Billex & Cambio de divisas & $6 \mathrm{G}$
\end{tabular}

Fuente: elaboración propia.

\subsection{Obtención de la información}

A partir de la revisión de la literatura y de entrevistas exploratorias a expertos en el tema, se elaboraron dos guías de entrevista semiestructurada dirigidas a los fundadores o gerentes de las fintech; la primera se enfocó en el proceso de innovación de las fintech y la segunda en el contexto de la organización. Luego, para validar los hallazgos, se elaboró una guía de entrevistas a expertos en temas de fintech e innovación. Las entrevistas se realizaron entre junio y julio de 2019 (ver tabla 2). Además, para complementar los datos de las entrevistas, se realizó una búsqueda exhaustiva de información en las páginas web oficiales de las fintech y en documentos internos compartidos por estas.

Tabla 2. Lista de entrevistados

\begin{tabular}{l|l|l|l} 
Código & Cargo e institución & Fecha de entrevista & Duración \\
\hline A1 & Fundador y gerente de operaciones Kambista & 18 de junio de 2019 & 50 minutos \\
\hline A2 & Country manager Perú Solven & $\begin{array}{l}20 \text { de junio de } 2019 \\
9 \text { de julio de } 2019\end{array}$ & $\begin{array}{l}60 \text { minutos } \\
35 \text { minutos }\end{array}$ \\
\hline
\end{tabular}




\begin{tabular}{|c|c|c|c|}
\hline A3 & Fundador y CEO Comparabien & $\begin{array}{l}25 \text { de junio de } 2019 \\
5 \text { de julio de } 2019\end{array}$ & $\begin{array}{l}50 \text { minutos } \\
42 \text { minutos }\end{array}$ \\
\hline A4 & Fundador y gerente comercial Innova Factoring & 27 de junio de 2019 & 46 minutos \\
\hline A5 & Fundador, COO y CTO Innova Factoring & 11 de julio de 2019 & 40 minutos \\
\hline A6 & Fundador y CFO Latin FinTech & $\begin{array}{l}10 \text { de julio de } 2019 \\
18 \text { de julio de } 2019\end{array}$ & $\begin{array}{l}43 \text { minutos } \\
30 \text { minutos }\end{array}$ \\
\hline A7 & Fundador y CEO Billex & 9 de julio de 2019 & 42 minutos \\
\hline A8 & $\begin{array}{l}\text { Encargado de alianzas en la Asociación Fintech Perú } \\
\text { y CEO de Kambista }\end{array}$ & 18 de julio de 2019 & 45 minutos \\
\hline A9 & Líder de disciplina de retail product BBVA & 17 de julio de 2019 & 30 minutos \\
\hline A10 & $\begin{array}{l}\text { Director fundador y consultor principal en innovación } \\
\text { y marketing en Galeas Jupiter Consulting y docente } \\
\text { PUCP }\end{array}$ & 19 de julio de 2019 & 57 minutos \\
\hline A11 & Monitor de proyectos Innóvate Perú & 19 de julio de 2019 & 56 minutos \\
\hline A12 & Especialista en open innovation BBVA & 19 de julio de 2019 & 36 minutos \\
\hline A13 & $\begin{array}{l}\text { Presidente Emprende UP y miembro del Consejo } \\
\text { Directivo de Fintech IberoAmérica }\end{array}$ & 22 de julio de 2019 & 47 minutos \\
\hline A14 & Director Innóvate Perú & 22 de julio de 2019 & 50 minutos \\
\hline
\end{tabular}

Fuente: elaboración propia.

\subsection{Proceso de análisis de la información}

Para el procesamiento y análisis de la información se siguió el método del análisis documental y se utilizó la herramienta ATLAS.ti. Primero, se determinaron los códigos y categorías de las variables de estudio: los habilitadores y las prácticas claves de la gestión del proceso de innovación. Luego, se procedió a la codificación de las entrevistas, para lo que se siguió la regla de frecuencia de Andréu (2002), la cual indica que de acuerdo a la cantidad de veces que aparecen nuevas variables en el trabajo de campo, se van generando códigos. Cabe resaltar que el proceso de codificación fue realizado por dos investigadores para comprobar la fiabilidad de la codificación y reducir el sesgo. Finalmente, se elaboraron las inferencias del objeto de estudio.

\section{Resultados y discusión}

\subsection{Proceso de innovación de las fintech seleccionadas}

Por su misma naturaleza, las fintech seleccionadas cuentan con un servicio innovador; sin embargo, dado que el objetivo de esta investigación es analizar cómo gestionan sus procesos de innovación, se consideró en el análisis solo aquellas innovaciones posteriores al lanzamiento de su servicio inicial, demostrando así que las fintech seleccionadas innovan continuamente.

Se encontró que la mayoría fintech seleccionadas (cinco de seis) realizan innovaciones de producto o servicio y de proceso, la mitad realiza innovaciones de 
mercadotecnia, y en ningún caso se presentó innovación organizacional. Asimismo, hay que resaltar que en todos los casos se trata de innovaciones incrementales, ya que son pequeñas mejoras a servicios, procesos o formas de comercialización ya existentes en el mercado.

Ahora bien, dado que todas las fintech estudiadas innovan continuamente, estas cuentan con un proceso de innovación; no obstante, solo cuatro de las seis fintech - Comparabien, Solven, Latin FinTech y Kambista - tienen este proceso sistematizado y, a excepción de Latin FinTech, realizan mediciones de los resultados de sus innovaciones.

Los entrevistados de las fintech resaltaron que la importancia de contar con un proceso de innovación sistematizado radica en contar con mayor orden y alinear las innovaciones a los objetivos de la organización. De la misma forma, la líder de retail product del BBVA explica que un proceso de innovación sistematizado debe ayudar a la fintech a ordenarse, pero debe ser flexible y sin burocracias ya que el diferenciador de las fintech es ser ágiles (A9). Asimismo, uno de los miembros de FinTech Iberoamérica afirma que todas las fintech tienen un proceso de innovación ya que su modelo de negocio así lo exige, y resalta que lo importante es que este proceso esté sistematizado (A13).

Por otro lado, respecto al grado de generación de ideas y el grado de implementación de estas, un experto en temas de innovación indica que una organización no solo tiene que tener la habilidad de generar ideas innovadoras, sino también la habilidad de ejecutarlas para poder generar innovación (A10). Por ello, se midió la efectividad del proceso de innovación de las fintech estudiadas en base al porcentaje de buenas ideas de nuevos servicios que se llegaron a lanzar al mercado en el periodo de un año. Como resultado, se obtuvo que la mayoría de las fintech no realiza un conteo de las ideas que genera y se enfoca en aquellas que llega a implementar; sin embargo, en base a los números aproximados que brindaron, se obtuvo que hay en promedio un 35\% de efectividad en el proceso de innovación, resaltando ComparaBien y Solven como las fintech con mayor efectividad con un $50 \%$.

Finalmente, se midió el tiempo promedio que les toma a las fintech estudiadas realizar el proceso de innovación para el lanzamiento de un nuevo servicio y se obtuvo lo siguiente: para la etapa de diseño y análisis, dos semanas; para la etapa de desarrollo, dos meses y medio; y, para la etapa de lanzamiento, dos semanas. Esto da un tiempo promedio total de tres meses y medio para todo el proceso de innovación. Al respecto, la líder de retail product del BBVA indicó que, en comparación con los bancos, las fintech son mucho más rápidas al momento de implementar un nuevo servicio (A9).

\subsection{Habilitadores del proceso de innovación}

Como se detalló líneas arriba, la gestión del proceso de innovación de las fintech estudiadas se analizó en base al modelo de Johnson et al. (2000). De esta forma, primero se identifican los habilitadores del proceso de innovación y luego las prácticas claves de cada etapa del modelo (ver figura 2) en las fintech estudiadas. 

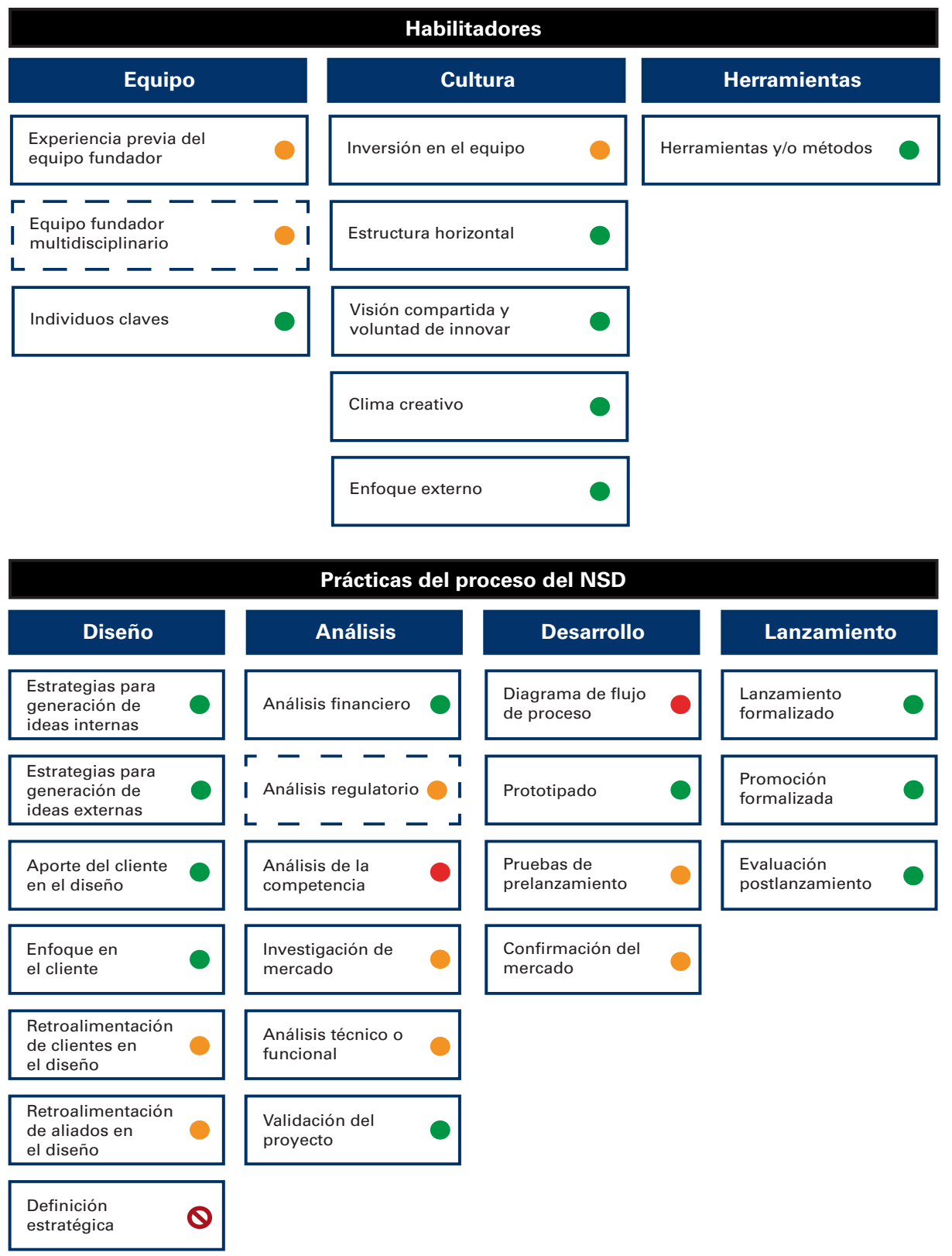

Leyenda: $\\lrcorner$ Habilitadores o prácticas clave encontradas en el trabajo de campo

$\square$ Habilitadores o prácticas clave que coinciden con la teoría

Q Habilitadores o prácticas clave que no coinciden con la teoría

Seis fintech la realizan

Cinco o cuatro fintech la realizan

Tres o menos de tres fintech la realizan 


\subsubsection{Equipo}

\section{a. Experiencia previa del equipo fundador}

La experiencia previa hace alusión a que en el equipo fundador al menos uno de los miembros cuente con experiencia en el sector financiero. Se encontró que en cinco de seis fintech estudiadas hay al menos un fundador con experiencia previa en instituciones financieras y ello resultó importante en la generación e implementación de innovaciones dentro de la organización. Al respecto, el director de Emprende UP indica que este habilitador es el más importante dentro de la categoría de equipo ya que, en un estudio previo realizado por Emprende UP para el Lima FinTech Fórum 2019, se encontró que la generación X (40 a 54 años) ocupa puestos gerenciales en más del $47 \%$ de las fintech registradas en SUNAT, y una característica de esta generación es que cuentan con experiencia previa en el sector (A13). En base a lo expuesto, este habilitador puede ser considerado como clave en el proceso de innovación de las fintech.

\section{b. Equipo fundador multidisciplinario}

El equipo fundador multidisciplinario es un habilitador que se encontró durante la fase de trabajo de campo, y fueron los entrevistados quienes enfatizaron este tema, ya que consideran que contar con un equipo fundador multidisciplinario es beneficioso en el desarrollo de sus procesos de innovación. En el caso de Comparabien, si bien hay un único fundador, él resaltó la importancia de contar con equipos multidisciplinarios y enfocados en una estrategia común para el éxito del proceso de innovación (A3). De igual forma, el director de Innóvate Perú afirma que «toda startup debe estar caracterizada por perfiles distintos, es esta la ventaja de las que ya están en el mercado» (A14). De esta forma, se deduce que contar con un equipo fundador multidisciplinario, como se aprecia en la mayoría de fintech estudiadas, puede aportar en la sistematización del proceso de innovación de las fintech, ya que, al agrupar diferentes perspectivas tanto del lado financiero como del lado tecnológico o incluso comercial, genera una combinación ideal no solo para la creación de la fintech, sino para la innovación continua en la organización.

\section{c. Individuos clave}

Respecto a los individuos clave, este habilitador se refiere a la existencia de roles que facilitan la innovación dentro de la organización (Froehle \& Roth, 2009). Se encontró que estos roles no están formalmente definidos en las fintech estudiadas; sin embargo, cuando surge una idea innovadora, resalta el rol de los gerentes como "patrocinadores", ya que motivan al equipo a ejecutar la idea; y el rol del equipo técnico como «campeones técnicos», pues se encargan de brindar soporte tecnológico. Además, los entrevistados resaltaron la importancia de que cada individuo del equipo tenga un "espíritu startup o espíritu de innovación», ya que, al tratarse de organizaciones de no más de 20 trabajadores y que no cuentan con área o persona encargada de la innovación, esta se convierte en tarea de todos. 
Por otro lado, los entrevistados de Latin FinTech y Solven resaltaron la importancia de que el equipo esté alineado a la cultura de la fintech para el éxito del proceso de innovación (A6 y A2). En síntesis, si bien en las fintech estudiadas no existen roles de innovación definidos formalmente, como lo indica la teoría, estos roles aparecen inconscientemente cuando surge una innovación a ser implementada y pueden ser asumidos por cualquier miembro de la organización en tanto cuentan con espíritu de innovación. Por ello, este habilitador se presenta en las fintech estudiadas como «individuos clave que tengan un espíritu de innovación».

\subsubsection{Cultura organizacional}

\section{a. Inversión en el equipo}

La inversión en el equipo hace alusión a que la fintech invierta en capacitaciones en temas de innovación. Se encontraron cuatro fintech que invierten en capacitaciones sobre temas de innovación y dos de ellas consideran importantes las capacitaciones en metodologías ágiles. En contraste, aquellas fintech que no invierten en este tipo de capacitaciones afirman que es porque "dan autonomía a sus colaboradores en este aspecto»(A2); o porque, "cuando nació la fintech, ya se habían brindado estas capacitaciones» (A4). El director de Emprende UP afirma que «el aprendizaje constante o la capacitación constante de cada uno de los diferentes roles motiva a la gente a quedarse contigo como también hace que se sistematicen las cosas en innovación» (A8). Es así que la inversión en el equipo puede considerarse un habilitador del proceso de innovación de las fintech seleccionadas.

\section{b. Estructura apropiada}

Respecto a la estructura apropiada, según los arquetipos estructurales de Mintzberg, la estructura simple es la más apropiada para la innovación en las fintech, ya que esta estructura se caracteriza por ser de tipo orgánico centralizado y porque puede responder rápidamente a los cambios en el medio ambiente (Tidd \& Bessant, 2009). Se encontró que la mayoría de fintech (cinco de seis) tiene una estructura simple que incentiva la innovación puesto que permite una comunicación horizontal. Solo en ComparaBien, su CEO considera que la estructura no influye en sus procesos de innovación; sin embargo, afirma que en un futuro sí impactará porque «espera tener a una persona encargada de gestionar el producto y la innovación» (A3). Para el presidente de Emprende UP la estructura horizontal o comunicación horizontal es considerada la variable de cultura organizacional más importante, ya que esta hace que el proceso de innovación sea más ágil (A13). Así, queda demostrado que contar con una estructura horizontal es un habilitador del proceso de innovación de las fintech seleccionadas.

\section{c. Visión compartida y voluntad de innovar}

La voluntad de innovar hace alusión a que la gerencia tenga una intención estratégica de innovar, mientras que la visión compartida hace referencia a que esta estrategia sea 
compartida a todos los miembros de la organización (Tidd \& Bessant, 2009). Respecto a la voluntad de innovar, todas las fintech indicaron que la innovación es una de sus principales estrategias, ya que el modelo de negocio de una fintech así lo exige y deben innovar continuamente para mantener su competitividad en el mercado y continuar creciendo. Expertos en el tema afirman que la gerencia debe ser quien impulse y apueste por la innovación para que esta se dé en la organización (A8 y A9). Además, se identificó que Innova Factoring, Comparabien y Solven consideran que la innovación es parte de su ADN en tanto no es una obligación, sino algo que hacen todos los días. Al respecto, el director de Emprende UP afirma que «las fintech nacen con un proceso de innovación, sino no pueden nacer, por lo tanto, la innovación es parte de su ADN» (A13).

En cuanto a la visión compartida, el country manager Perú de Solven afirma que «el equipo tiene que estar comprometido y alineado culturalmente con la startup y el ideal del fundador» (A2). A partir de lo expuesto, queda claro que la innovación no solo es parte de la estrategia de las fintech, sino que es parte de la misma fintech; es decir, es algo que hacen todos los días y que, por ende, está inmerso en su cultura. En conclusión, la visión compartida y la voluntad de innovar pueden considerarse un habilitador del proceso de innovación de las fintech estudiadas.

\section{d. Clima creativo}

El clima creativo hace alusión a que exista un enfoque positivo a las nuevas ideas innovadoras que provengan de cualquier miembro de la organización (Tidd \& Bessant, 2009). Sobre este habilitador, los entrevistados comentaron que brinda apoyo y espacio a sus equipos para la generación de ideas. Este hallazgo es importante pues esta "cultura de opinión abierta» va de la mano con la estructura simple de las fintech, la cual, como se explicó líneas arriba, permite una comunicación horizontal entre todos los miembros de la organización.

Por otro lado, un tema resaltante mencionado por los entrevistados es la importancia de la "tolerancia al fracaso». EI CEO de Kambista afirma que "la clave para fomentar la innovación dentro de la organización es desarrollar una cultura organizacional que sea tolerante al fracaso» (A8). De la misma forma, la líder de disciplina de retail product del BBVA afirma que las fintech deben promover "una cultura de aceptar el error, aceptar el fracaso y revisar los errores ya que ello ayuda mucho a la innovación» (A9). De esta forma, un "clima creativo con tolerancia al fracaso» puede ser considerado como un habilitador del proceso de innovación de las fintech seleccionadas.

\section{e. Enfoque externo}

El enfoque externo se refiere a que la organización tenga una amplia red de contactos o cuente con alianzas estratégicas que influyan en sus procesos de innovación (Tidd \& Bessant, 2009). Sobre este habilitador, los entrevistados consideran que es importante trabajar con universidades, incubadoras o aceleradoras ya que estas alianzas les permiten mantenerse continuamente capacitados e informados de los últimos estudios o análisis tecnológicos sobre innovación. Además, consideran relevante mantener contacto con otras 
fintech o instituciones financieras pues ello les permite estar al tanto de las tendencias del sector y les abre la posibilidad de generar alianzas estratégicas para innovar.

Por otro lado, todas las fintech, a excepción de Comparabien, forman parte de una de las dos asociaciones fintech peruanas y consideran que pertenecer a una asociación "da lineamientos a los actores del ecosistema que quieren hacer innovación y permite que puedan colaborar mejor» (A1). Al respecto, el presidente de Emprende UP afirma que las incubadoras o centros de emprendimiento son las organizaciones que más influyen en el proceso de innovación de las fintech puesto que son especialistas en el tema de fintech (A13). Asimismo, un estudio empírico previo resaltó que las fintech realizan innovación de servicios en colaboración con múltiples socios en un entorno empresarial y ecosistema cambiante (Riikkinen et al., 2019). Por todo lo expuesto, el enfoque externo puede considerarse un habilitador del proceso de innovación.

\subsubsection{Herramientas}

\section{a. Herramientas}

El uso de herramientas es el último habilitador identificado del proceso de innovación. A continuación, se explicarán al detalle las herramientas de gestión utilizadas por las fintech estudiadas en cada etapa del ciclo de proceso del NSD. Al respecto, se debe tomar en cuenta que algunas herramientas pueden ser utilizadas en más de una fase del modelo de innovación.

\section{Herramientas utilizadas durante el diseño}

En cuanto a la generación de ideas, la herramienta más utilizada por todas las fintech estudiadas es el brainstorming debido a su practicidad y facilidad de implementación. Además, en Comparabien realizan un análisis de causa raíz para que las ideas "sean direccionadas y para que no sea tan abierto como el brainstorming» (A3). Por otro lado, la mayoría de fintech (cinco de seis) utiliza las encuestas de satisfacción del cliente. Respecto a las herramientas para el desarrollo de concepto, destaca el uso del design thinking por parte de cinco de las seis fintech seleccionadas. Al respecto, se encontró que estas empatizan con sus clientes, lo cual es un input para la fase de definición e ideación; realizan prototipos del nuevo servicio (de dos hasta cinco); y, a excepción de Latin FinTech, realizan el testeo o confirmación de mercado previo al lanzamiento; todo lo cual demuestra que efectivamente estas fintech aplican los conceptos básicos del design thinking. Sobre este hallazgo, los expertos indican que "el design thinking es una herramienta que les permite identificar cuál es su mercado" (A14) y "te permite prototipar y siempre preguntar primero a tus clientes, algo que a veces le falta realizar a los bancos» (A9). Otras herramientas son el service blueprint, utilizado por Solven e Innova Factoring pues les permite conocer el servicio desde la perspectiva del cliente (A5); el journey map, utilizado por Billex porque les permite identificar fácilmente los puntos de mejora del proceso; y, las pruebas de concepto, utilizadas por Billex y Latin FinTech mediante encuestas a clientes o entrevistas presenciales, lo cual les permite afinar el diseño del nuevo servicio. 


\section{Herramientas utilizadas durante el análisis}

Respecto a la etapa de análisis, las herramientas que las fintech estudiadas utilizan son en su mayoría herramientas de ofimática. Además, en Solven utilizan Power BI ya que facilita el análisis predictivo de la data existente y facilita la toma de decisiones. Por otro lado, el gerente de operaciones de Kambista indicó que utilizan una matriz de impacto y viabilidad, ya que les permite identificar fácilmente aquellas innovaciones que implican menos esfuerzo y mayor impacto (A1).

\section{Herramientas utilizadas durante el desarrollo}

En la etapa de desarrollo en general destaca el uso del Scrum, el cual es un marco de trabajo para el desarrollo ágil y el mantenimiento de productos complejos (Sutherland, 2010). La líder de retail product del BBVA comenta que el Scrum permite tener al equipo concentrado en un proyecto, lo que ayuda a terminarlo más rápido (A9).

Asimismo, Innova Factoring, Solven y Latin FinTech aplican algunos conceptos de Lean Startup, no solo en la etapa de desarrollo, sino en su proceso de innovación en general. Al respecto, los expertos indican que el Lean Startup «es una herramienta donde desarrollas productos mínimos viables, usas el Canvas y, a través de experimentos, evalúas los resultados del mercado y tomas decisiones» (A10).

Por otro lado, en cuanto a las herramientas utilizadas para las pruebas de prelanzamiento, cuatro de las seis fintech estudiadas indicaron utilizar Unit Test, zonas de prueba y herramientas inhouse. Sobre estas se encontró que si bien las herramientas para las pruebas prelanzamiento son importantes, estas son costosas y toman tiempo, lo cual puede ser un motivo por el que no todas las fintech realizan dichas pruebas. Finalmente, respecto a la confirmación del mercado, dos fintech indicaron utilizar el $A / B$ testing por su calidad de confirmación previa al lanzamiento de un nuevo servicio al mercado; y tanto Solven como Latin FinTech indican que realizan el testeo mediante el lanzamiento de un MVP a una muestra pequeña, y que van mejorando el nuevo servicio en base a las sugerencias que sus clientes les dan.

\section{Herramientas de lanzamiento}

En el lanzamiento, todas las fintech indicaron utilizar herramientas propias de los canales digitales que les permiten contar con métricas de respuesta de la publicidad. Por otro lado, respecto a la evaluación postlanzamiento, en el caso de Comparabien su CEO indicó que utilizan herramientas específicas como Amplitud, que permite hacer seguimiento de métricas de tiempo de utilización de la plataforma (A3); y en Solven su country manager Perú indicó que utilizan Pipedrive porque les permite hacer un CRM (Customer Relationship Management) (A2). Además, las demás fintech indicaron que realizan encuestas a clientes o llamadas telefónicas para conocer la percepción de sus clientes en relación con el nuevo servicio lanzado.

En general, un hallazgo importante sobre el habilitador de herramientas es el uso de metodologías ágiles en las diferentes etapas del proceso de innovación de las 
fintech estudiadas, lo cual encaja con lo encontrado en un reciente estudio sobre fintech en Finlandia (Riikkinen et al., 2019). Al respecto, expertos en fintech afirman que no es solo implementar una metodología ágil porque sea tendencia, sino elegir las que mejor se adapten al modelo de negocio de la fintech y tropicalizarla al contexto (A12 y A8). Asimismo, el director de Innóvate Perú afirma que «si una fintech hoy en día no tiene metodologías ágiles no va a poder adoptar tecnologías nuevas porque están diseñadas para que sean usadas con metodologías ágiles» (A14).

Sobre las tecnologías, se encontró que aquellas que utilizan o piensan utilizar las fintech estudiadas son: las API (Interfaz de Programación de Aplicaciones), el Big Data, el Blockchain e Identidad Digital. Al respecto, un estudio previo afirma que las tecnologías desempeñan un papel habilitador y aseguran que el proceso de creación de valor colaborativo en la innovación del servicio sea eficiente y eficaz (Riikkinen et al., 2019); además, facilitan la interacción entre los actores y recursos (Lusch \& Nambisan, 2015).

En definitiva, podemos afirmar que el uso de herramientas de gestión y metodologías ágiles durante el proceso de innovación de servicios de las fintech estudiadas son habilitadores clave, ya que agiliza el proceso y les permite adoptar nuevas tecnologías de forma eficiente.

\subsection{Principales prácticas del proceso de innovación}

A continuación, se detallarán las prácticas clave encontradas en las fintech seleccionadas en base a cada etapa del modelo de Johnson et al. (2000).

\subsubsection{Diseño}

\section{a. Estrategias de generación de ideas}

Las estrategias de generación de ideas son aquellas relacionadas al uso de fuentes internas y externas para generar ideas innovadoras. Al respecto, todos los entrevistados indicaron que los empleados son la principal fuente interna de generación de ideas, lo cual está relacionado con una "cultura de opinión abierta» y una "comunicación horizontal»; es decir, los habilitadores de clima creativo y estructura apropiada tienen una relación directa con esta práctica.

Por otro lado, la generación de ideas a través de fuentes externas en las fintech estudiadas se da gracias al aporte de los clientes, la consulta directa a otras fintech o la revisión de las tendencias que existen en el mercado. De la misma forma, en un estudio reciente sobre innovación de servicios digitales en fintech se identificó que diferentes actores del ecosistema del servicio como clientes, proveedores, empleados, etc., pueden aportar y propiciar cambios al proceso de creación de valor de los servicios (Riikkinen et al., 2019). Al respecto, uno de los expertos afirma que las fintech deben estar al tanto de las ideas de mercados internacionales con miras a poder aterrizarlas al mercado local y generar nuevos servicios innovadores (A12). En síntesis, el uso de estrategias de generación de ideas es una práctica clave en la etapa de diseño y esta se encuentra potenciada por el clima creativo y una estructura horizontal. Además, se identificó que el surgimiento de ideas 
innovadoras del nuevo servicio puede darse en cualquier fase del proceso de innovación y, por consiguiente, generar un reproceso o añadir mejoras incrementales a un nuevo servicio ya diseñado.

\section{b. Involucramiento del cliente en el diseño}

El involucramiento del cliente en el diseño se conforma por las siguientes prácticas: aporte del cliente en el diseño, enfoque en el cliente y retroalimentación del concepto del cliente. La práctica de aporte del cliente en el diseño es desarrollada por las fintech estudiadas cuando recopilan información y feedback de sus clientes mediante encuestas de satisfacción, redes sociales o explotación de data de clientes para diseñar nuevos servicios enfocados en satisfacer sus necesidades. Al respecto, la experta en open innovation del BBVA menciona que "las fintech tienen la ventaja de estar cerca al cliente y poder conocerlo al 100\%, algo que les cuesta mucho a las instituciones financieras» (A12). Es así que esta práctica se considera característica del proceso de innovación de las fintech estudiadas.

Respecto al enfoque en el cliente, los entrevistados mencionaron que buscar satisfacer a sus clientes es una práctica que guía todas las actividades que realizan, incluso mencionaron que puede considerarse una ventaja competitiva ya que, a diferencia de los bancos, esta práctica les permite asegurarse de entregar valor a sus clientes y, además, generar fidelización en el servicio (A1). Estos hallazgos coinciden con los resultados de un estudio reciente que afirma que las innovaciones en fintech se basan en la comprensión del cliente para ofrecer valor de una manera novedosa (Riikkinen et al., 2019). De esta forma, se concluye que el enfoque en el cliente es una práctica clave en la etapa de diseño.

La retroalimentación del cliente en el diseño consiste en que la organización considere comentarios de los clientes sobre el concepto del nuevo servicio ya formulado para poder tener el último feedback previo a la fase del desarrollo formal (Tidd \& Bessant, 2009). Al respecto, los entrevistados de las fintech (cuatro de seis) mencionaron que la retroalimentación del cliente en el diseño no solo se da previo al desarrollo formal, sino también después de ya haber implementado el nuevo servicio. Según palabras del CEO de Billex: "los clientes nos dan sugerencias sobre lo que ya vamos implementando en la segunda versión de la plataforma» (A7). Asimismo, el CEO de Kambista menciona que la retroalimentación de los clientes «es clave y evidencia que el único jefe de una fintech es el cliente ya que el servicio que ofrecen obedece a sus expectativas, opiniones y requerimientos» (A8). En síntesis, se concluye que estas tres prácticas se relacionan directamente entre ellas; es decir, una fintech buscará información del cliente en el diseño del servicio con data obtenida de retroalimentaciones o encuestas.

\section{c. Involucramiento de aliados en el diseño}

La retroalimentación de aliados en el diseño es una práctica desarrollada por cuatro de las seis fintech estudiadas pues estas consideran los comentarios de socios o proveedores durante la conceptualización de la idea del servicio. Para el CEO de Kambista el feedback de los proveedores y aliados es clave en el desarrollo del concepto (A8), y se identificó que son los proveedores quienes suelen cumplir el rol de facilitadores tecnológicos en el proceso 
de innovación. Por todo lo expuesto, se afirma que esta es una práctica clave del proceso de innovación de las fintech estudiadas.

\section{d. Definición estratégica}

Respecto con la definición estratégica de objetivos para el nuevo servicio, los entrevistados mencionaron que estos deben estar alineados a los objetivos estratégicos de la organización. Los entrevistados de Comparabien, Solven y Latin FinTech consideran importante que el nuevo servicio a desarrollar esté alineado a sus objetivos estratégicos, misión y visión. En resumen, los entrevistados consideran muy importante que los objetivos del nuevo servicio estén alineados a los objetivos estratégicos de la organización; sin embargo, no se especifican objetivos propios del nuevo servicio. Por lo tanto, esta práctica encontrada en la teoría no se aplica por completo en las fintech estudiadas y no puede considerarse como práctica clave en el proceso de innovación.

\subsubsection{Análisis}

\section{a. Análisis financiero}

El análisis financiero es una práctica realizada por todas las fintech estudiadas y se caracteriza por ser simple, rápido y de corto plazo. Además, se identificó que las fintech no establecen un rendimiento económico a largo plazo, sino que se basan en proyecciones a un año como máximo. Asimismo, señalan que este análisis les sirve como input para evaluar la viabilidad del desarrollo de un nuevo servicio, así como buscar inversionistas que financien sus innovaciones.

Uno de los expertos en innovación explica que para las startup (fintech) el análisis de la parte financiera es muy simple y que utilizan algunos indicadores como the lifetime value o el churn rate (A10). En síntesis, esta práctica en las fintech, al igual que indica la teoría, es necesaria en la fase inicial o de planeamiento del proceso de innovación y basa el control económico de gasto de recursos que tendrá el nuevo servicio al ser implementado.

\section{b. Análisis regulatorio}

El análisis regulatorio es una práctica hallada en campo, pues cuatro de las seis fintech entrevistadas reconocieron el papel de la legislación dentro de sus actividades en tanto realizan este análisis previo al desarrollo o lanzamiento de un nuevo servicio. Se encontró que para las fintech el tema regulatorio podría ser un nuevo camino de desarrollo para innovaciones que mejoren sus procesos o ser un obstáculo debido a la falta de claridad regulatoria. Sobre esto, el presidente de Emprende UP indica que las fintech deben pensar en la regulación incluso antes del lanzamiento porque después de este será difícil solucionarlo (A13). Además, el experto afirmó que a la fecha hay regulaciones que están por salir como es el caso de la ley de crowdfunding o financiamiento participativo, la cual va a impactar directamente en las fintech de este segmento, que tendrán que adecuarse y mejorar su modelo de negocio (A13). En síntesis, los cambios en el mercado a menudo 
están respaldados por la regulación, lo que significa que los recién llegados pueden crear sus servicios directamente de acuerdo con las reglas existentes en aquel momento, mientras que el resto de las fintech tendrán que amoldarse a estas.

\section{c. Análisis del mercado}

Respecto al análisis del mercado, este se conforma por dos prácticas: investigación de mercado y análisis de la competencia. La práctica de investigación de mercado es realizada por cinco de las seis fintech estudiadas y consiste en ver qué tan viable es el nuevo servicio; es decir, si este responde a las expectativas de los clientes, de la propia fintech o al nivel de crecimiento esperado. La líder de retail product del BBVA recomienda que las fintech tengan ordenada o estructurada la información del mercado, y establecer tiempos para la recolección de dicha información debido a que un nicho de mercado puede sufrir cambios inesperados (A9). Sin embargo, el presidente de Emprende UP menciona que el análisis de mercado suele ser una práctica característica de los bancos, más no de las fintech (A13). En este sentido, podemos afirmar que realizar una investigación de mercado puede resultar útil para las fintech y es una práctica clave en la etapa de análisis.

Por otro lado, el análisis de la competencia es realizado por solo dos de las fintech seleccionadas. Además, según el presidente de Emprende UP, las fintech no poseen información accesible para hacer un análisis de competencia porque la información es restringida (A13). Por ello, se considera que el análisis de la competencia es una práctica que no es característica del proceso de innovación.

\section{d. Análisis funcional}

El análisis funcional es realizado por cinco de las seis fintech estudiadas para conocer la viabilidad del nuevo servicio a desarrollar. Al respecto, el director de Innóvate Perú opina que las fintech no deberían enfocarse mucho en registrar los análisis que realizan, ya que les demandaría demasiado tiempo; en cambio, recomienda contar con herramientas tecnológicas que les permitan realizar este análisis de forma natural y rápida (A14). En este sentido, esta práctica se considera clave en la etapa de análisis y debe ser facilitada por el uso de herramientas.

\section{e. Validación del proyecto}

La práctica de validación del proyecto es realizada por todas las fintech, siendo los criterios más relevantes —evaluados por la mayoría de fintech estudiadas - el retorno financiero, seguido por la experiencia brindada al usuario y la escalabilidad. Asimismo, las personas relacionadas con la generación y evaluación de estos criterios son los fundadores o CEO en reunión con las diferentes gerencias. Al respecto, uno de los expertos opina que la toma de decisiones, al igual que las demás prácticas, debe ser flexible y no tan burocrática para que se realice de forma rápida y ágil (A9). A partir de lo expuesto, validar el nuevo servicio previamente a su desarrollo permite conocer el impacto que este tendría y puede considerarse una práctica clave del proceso de innovación. 


\subsubsection{Desarrollo}

\section{a. Diagrama de flujo de proceso}

El diagrama de flujo de proceso es la práctica menos utilizada en la fase de desarrollo, pues solo tres de las seis fintech estudiadas realizan un service blueprint o journey map del nuevo servicio a ofrecer. Innova Factoring y Solven utilizan el service blueprint en sus procesos, y Billex utilizó un journey map — con el apoyo de su proveedor NovitLab_ para el desarrollo de su nueva plataforma de servicio (Novitlab, 2019). Pese a que esta práctica es poco utilizada, el director de Emprende UP indica que es la más importante dentro de la etapa de desarrollo, ya que permite mapear el servicio que se está ofreciendo a los clientes e identificar a todos los intervinientes, las variables, los puntos de dolor y los procesos a seguir (A13).

\section{b. Prototipado}

El prototipado es la principal práctica utilizada por todas las fintech estudiadas porque les permite acercarse al servicio funcional que desean brindar, además de permitirles evaluar las partes vitales del nuevo servicio antes de invertir sus recursos en ello. Asimismo, se halló que las fintech desarrollan de dos a cinco MVP (mínimo producto viable, en español). De acuerdo con la teoría, esta es una de las prácticas más utilizadas (Froehle \& Roth, 2009) para probar nuevos procesos o sistemas de servicios (Edvardsson \& Olsson, 1996; Bitran \& Pedrosa, 1998). Al respecto, el CEO de Kambista indicó que el prototipado es una práctica clave ya que es donde uno ejecuta y tangibiliza lo planeado (A8). Además, en un estudio sobre innovación en diez fintech de Finlandia se identificó que la elaboración de prototipos es parte de la pila de innovación de servicios de las fintech (Riikkinen et al., 2019). Por todo lo expuesto, realizar prototipos se considera una práctica clave del proceso de innovación.

\section{c. Pruebas de prelanzamiento}

La práctica de prelanzamiento hace referencia a la realización de las pruebas de calidad antes del lanzamiento (Froehle \& Roth, 2009). Se encontró que cuatro de las seis fintech estudiadas realizan estas pruebas porque les permite asegurarse de contar con un estándar mínimo de calidad de su nuevo servicio para que pueda competir en el mercado. Contrario a esto, el director de Emprende UP considera que invertir demasiado tiempo en esta actividad generaría retrasos en sus procesos, lo que ocasionaría que las fintech ya no sean ágiles (A13). De igual manera, el country manager Perú de Solven considera que este procedimiento, si bien es crítico, no es indispensable para una startup, ya que demanda una gran cantidad de tiempo (A2). Por lo tanto, esta práctica puede considerarse clave en la etapa de desarrollo, siempre y cuando no demande mucho tiempo.

\section{d. Confirmación del mercado}

Respecto a la confirmación del mercado, se encontró que cinco de las seis fintech seleccionadas generan pruebas de mercado con el fin de corroborar que el nuevo servicio 
sea comercializable y se haya desarrollado idóneamente. Los entrevistados indicaron este testeo les permite conocer de manera directa la respuesta de su público objetivo sobre el nuevo servicio a lanzar y corregir las posibles fallas, lo que reduce el riesgo de fracasar al momento de introducir el nuevo servicio al mercado. En Latin FinTech no se realizan estas pruebas de mercado ya que recogen feedback constante de terceros durante el diseño y consideran que no es necesario (A6). Sin embargo, para los expertos, la confirmación del mercado es la actividad más valiosa dentro del desarrollo puesto que reduce el riesgo de fracaso durante el lanzamiento y permite identificar lo que mejor funciona (A8 y A14). Por ello, esta práctica se considera clave en el desarrollo del nuevo servicio.

\subsubsection{Lanzamiento}

\section{a. Lanzamiento formalizado}

En cuanto al lanzamiento formalizado, se encontró que todas las fintech realizan un lanzamiento formalizado. Además, tres de las fintech estudiadas trabajan en conjunto con una agencia de marketing, lo cual les resulta ventajoso ya que estas agencias tienen una vasta experiencia en el rubro. Para el director de Innóvate Perú, la etapa de lanzamiento es estructurada para este tipo de organizaciones (A14). Asimismo, para uno de los miembros del consejo directivo de FinTech Iberoamérica, el lanzamiento formalizado es la actividad más importante de la etapa de lanzamiento (A13). Por todo lo expuesto, se considera que el lanzamiento formalizado es una práctica clave en el proceso de innovación de las fintech estudiadas.

\section{b. Promoción formalizada}

Respecto a la promoción formalizada, las fintech realizan esta práctica mediante canales digitales, utilizando redes sociales como Facebook, Instagram y Linkedln. Se encontró que cuatro de las seis fintech seleccionadas realizan un programa promocional detallado con la finalidad de comunicar, informar o recordar el nuevo servicio lanzado al mercado para así motivar o inducir el consumo de este. Además, se identificó que Comparabien no realiza un plan promocional pues el servicio que ofrecen es gratuito, por lo que no es necesario elaborarlo (A3). De esta forma, se afirma que la promoción formalizada es una práctica clave en el lanzamiento, dependiendo del tipo de servicio que se ofrezca.

\section{c. Evaluación postlanzamiento}

Sobre la evaluación postlanzamiento, se encontró que todas las fintech estudiadas realizan esta práctica para conocer la opinión de sus clientes respecto al nuevo servicio. Los medios más comunes utilizados por estas fintech para recopilar feedback de los clientes son las llamadas telefónicas, seguidas por las encuestas de satisfacción. Además, los entrevistados indicaron que la información que recopilan les ayuda a mejorar el servicio ofrecido, permitiéndoles un acercamiento a sus clientes a través de un intercambio de sugerencias y/o quejas para mejorar sus servicios. En definitiva, la evaluación postlanzamiento puede 
considerarse una práctica clave de la etapa final del proceso de innovación de las fintech estudiadas.

\section{Conclusiones}

En esta investigación, el objetivo fue analizar la gestión del proceso de innovación de seis fintech peruanas en base al modelo NSD de Johnson et al. (2000), identificando los habilitadores y prácticas claves característicos de las fintech estudiadas. Este estudio revela que las fintech analizadas innovan por la alta competitividad en el sector, la necesidad de crecer y, principalmente, porque su propio modelo de negocio así lo exige. Sin embargo, existen barreras que dificultan sus innovaciones como la falta de prioridad de parte de sus aliados, el acceso a financiamiento y la demora de la regulación respecto a nuevas tecnologías como la identidad digital.

Las innovaciones en servicio y procesos son el tipo de innovación que más realizan las fintech estudiadas, siendo la innovación en mercadotecnia la que menos realizan. Asimismo, en todos los casos se trata de innovaciones incrementales, lo cual demuestra que las fintech estudiadas innovan continuamente, incluso después de lanzar su servicio principal al mercado.

Respecto al proceso de innovación, este se encuentra sistematizado y estructurado solo en tres de las seis fintech estudiadas, las cuales realizan mediciones de los resultados de sus innovaciones. Sin embargo, se identificó que todas las fintech analizadas cuentan con habilitadores y prácticas clave del proceso de innovación, los cuales les permiten lanzar innovaciones al mercado de forma exitosa. Ello se ve reflejado en la efectividad de sus procesos de innovación, ya que en promedio tienen un 35\% de efectividad, medida en el porcentaje de ideas de nuevos servicios que llegan a lanzarse al mercado de forma exitosa. Además, el tiempo promedio de sus procesos de innovación es de tres meses y medio, demostrando que son más ágiles que los bancos.

En cuanto a los habilitadores que facilitan el proceso de innovación de las fintech analizadas, a nivel de equipo destacan: la experiencia previa del equipo fundador en el sector financiero, el equipo fundador multidisciplinario e individuos claves con espíritu de innovación. En cultura organizacional destacan: la estructura simple y horizontal, la visión compartida y la voluntad de innovar, el clima creativo con tolerancia al fracaso y el enfoque externo. $Y$, en cuanto al uso de métodos y/o herramientas de apoyo para el proceso de innovación, destacan: el brainstorming, el design thinking y el service blueprint o journey map para la etapa de diseño; el Scrum y las herramientas in-house de prototipado para la etapa de desarrollo; y, los canales digitales y encuestas a clientes en la etapa de lanzamiento. En general, sobre este último habilitador, destaca el uso de metodologías ágiles y la importancia de contar con tecnologías como las API, el Big Data, el Blockchain y la Identidad Digital.

Respecto a las prácticas claves del modelo de ciclo del proceso de NSD, se identificaron las siguientes prácticas en la fase de diseño: el uso de estrategias de generación de ideas utilizando fuentes internas —empleados- y externas —clientes, proveedores, fintech-, el aporte del cliente en el diseño y el enfoque del cliente, lo cual les permite diseñar nuevos servicios pensados en satisfacer las necesidades de sus clientes. 
En segundo lugar, en la fase de análisis, las principales prácticas realizadas por las fintech estudiadas son: el análisis financiero, caracterizado por ser simple, rápido y de corto plazo; y el análisis técnico, el cual busca conocer los recursos, la tecnología y la capacidad para desarrollar el nuevo servicio. Asimismo, se encontró en el campo que las fintech analizadas realizan un análisis regulatorio con el objetivo de aminorar los riesgos legales que trae consigo el desarrollo de una innovación. Además, respecto a la validación del proyecto, el principal criterio que toman en cuenta es el retorno financiero y la mejora de la experiencia de usuario, los cuales son validados a nivel gerencial.

En tercer lugar, en la fase de desarrollo se identificaron las siguientes prácticas claves: el diagrama de flujo de procesos para mapear el nuevo servicio e identificar posibles puntos de dolor; el prototipado para ejecutar y tangibilizar lo planeado; las pruebas prelanzamiento para permitir asegurar un estándar mínimo de calidad; y la confirmación de mercado para asegurar el desarrollo óptimo del nuevo servicio y así reducir los riesgos de fracaso antes del lanzamiento. En cuarto lugar, en la fase de lanzamiento se identificó que las fintech analizadas realizan las siguientes prácticas claves: lanzamiento formalizado, diseñado internamente o con agencias de marketing; promoción formalizada mediante canales digitales como redes sociales para comunicar el nuevo servicio; y evaluación postlanzamiento a través del uso de encuestas o llamadas telefónicas a clientes para conocer sus opiniones y así mejorar el servicio.

Por último, esta investigación es un avance en el conocimiento sobre la innovación de servicios en las fintech, lo cual resulta de interés para los CEO de las fintech, policy makers y académicos. Dado el creciente interés de los bancos en las fintech, este estudio les permitirá conocer cómo innovan estas nuevas empresas. Y, en el caso de las nuevas fintech, estas podrán conocer cuáles son los principales habilitadores y prácticas del proceso de innovación.

Sin embargo, pese a todo lo expuesto, este estudio posee algunas limitaciones que resulta necesario clarificar. En primer lugar, dado que las fintech analizadas son de diferentes segmentos y no son un número representativo del universo de fintech peruanas, los resultados obtenidos no se pueden extrapolar a todas las fintech del contexto peruano. En segundo lugar, sería provechoso realizar estudios que analicen a profundidad cómo se implementan cada uno de los principales habilitadores o prácticas claves del proceso de innovación y de qué forma impactan en el éxito del nuevo servicio. En tercer lugar, sería interesante continuar ampliando el conocimiento en este tema llevando a cabo investigaciones cuantitativas de tipo correlacional que demuestren y expliquen la relación existente entre la sistematización del proceso de innovación de una fintech y los resultados de la innovación, lo cual generaría una visión más completa sobre la importancia del proceso de innovación en estas organizaciones. En general, se invita a realizar más investigaciones sobre innovación en fintech, ya que estos nuevos actores del sistema financiero prometen cambiar la forma en que se brindan los servicios financieros en el mercado peruano. 


\section{bibliografía}

\section{Andréu, J.}

2002

Las técnicas de análisis de contenido: una

revisión actualizada. Sevilla: Fundación

Centro Estudios Andaluces.

\section{Barras, R.}

1990

Interactive innovation in financial and

business services: The vanguard of the

service revolution. Research Policy, 19(3),

215-237.

Benito, S., Platero, M., \&

Rodríguez, A.

Factores determinantes de la innovación en las microempresas españolas: la importancia de los factores internos. Universia Business Review, 33(1), 104-121.

\section{Banco Interamericano de Desarrollo [BID], BID Invest, \&} Finnovista.

FinTech América Latina 2018: Crecimiento y consolidación. doi: http://dx.doi.

org/10.18235/0001377

\section{Bilderbeek, R., Den Hertog, P.,} Marklund, G., \& Miles, I.

1998 Services in Innovation: Knowledge Intensive Business Services (KIBS) as CoProducers of Innovation. SI14S Synthesis Paper N 3. SI14S Project, STEP Group.

\section{Bitran, G., \& Pedrosa, L.}

A structured product development perspective for service operations. European Management Journal, 16(2), 169-189
Brady, T., Rush, H., Hobday, M., Davies, A., Probert, D., \& Banerjee, $\mathbf{S}$.

Tools for technology management: An academic perspective. Technovation, 17(8), 417-426

\section{Castro, E.}

2010 El estudio de casos como metodología de investigación y su importancia en la dirección y administración de empresas. Revista Nacional de Administración, 1(2), 31-54.

\section{Cowell, D.}

New service development. Journal of Marketing Management, 3(3), 296-312.

\section{Dodgson, M., Gann, D., \& Phillips, $\mathbf{N}$.}

Perspectives on Innovation Management. En M.Dodgson, D. Gann y N. Phillips (eds.), The Oxford Handbook of Innovation Management. Nueva York: Oxford University Press. doi: 10.1093/ oxfordhb/9780199694945.013.037

\section{Edvardsson, B., \& Olsson, S.}

Key concepts for new service development. The Service Industries Journal, 16(2), 140-164. doi: $10.1080 / 02642069600000019$

Eisenhardt, K. M., \& Graebner, M. E.

2007 Theory building from cases: Opportunities and challenges. Academy of Management Journal, 50(1), 25-32. 


\section{bibliografía}

Fernández, C.

2012

Determinantes de la capacidad de innovación es pymes regionales. Revista de Administração da Universidade Federal de Santa María, 5(1), 749-765.

\section{Freeman, C.}

Innovation change of techno-economic paradigm and biological analogies in economics. Review of Economics, 42(2), 211-232.

Froehle, C. M., \& Roth, A. V.

A Resource-Process Framework of New Service Development. Production and Operations Management, 16(2), 169-188. doi: 10.1111/j.1937-5956.2007.tb00174.x

Froehle, C. M., Roth, A. V., Chase, R. B., \& Voss, C. A.

2010

Antecedents of New Service Development Effectiveness: An Exploratory Examination of Strategic Operations Choice. Journal of Service Research, 3(1), 3-17.

\section{Fundación COTEC.}

Informe COTEC: tecnología e innovación

en España. Madrid: Fundación para la Innovación Tecnológica.

\section{Jack, E. P., \& Raturi, A. S.}

Lessons learned from methodological triangulation in management research. Management Research News, 29(6), 345-357.

\section{Johnson, S., Menor, L., Roth,}

\section{A., \& Chase, $R$.}

New Service Development: Creating Memorable Experiences (pp. 1-32). California: Sage Publications.

\section{Khraisha, T., \& Arthur, K.}

¿Can we have a general theory of financial innovation processes? A conceptual review. Financial Innovation, 4(4).

\section{Miles, I.}

Innovation in service. En J. Fagerberg, D. Mowery y R. Nelson (eds.), Oxford Handbook of Innovation. Oxford: Oxford University Press. doi: 10.1093/ oxfordhb/9780199286805.003.0016

\section{Ministerio de la Producción.}

Bases integradas del Concurso

Capital Semilla para Emprendimientos

Innovadores. Recuperado de: https://

innovateperu.gob.pe/fincyt/doc/

emprendimiento-innovador/7G/bases/

Bases_innovadores_integradas.pdf

Morales, M., Ortíz Riaga, C., \& Arias Cante, $M$.

Factores determinantes de los procesos de innovación: una mirada a la situación en Latinoamérica. Revista Escuela de Administración de Negocios, (72), 148-163.

\section{Lusch, R., \& Nambisan, S.}

2015

Service innovation: A service-dominant logic perspective. Management Information Systems Quarterly, 39(1), 155175. doi: 10.25300/MISQ/2015/39.1.07

\section{Nelson, R., \& Winter, S.}




\section{bibliografía}

Nicoletti, B.

2017 The Future of FinTech. Primera edición.

Roma: Palgrave Macmillan. doi:

10.1007/978-3-319-51415-4

\section{NovitLab.}

2019

UX de aplicación web para la operación de divisas. Recuperado de: http://novit.pe/ billex/

\section{Organización para la}

Cooperación y el Desarrollo

Económicos [OCDE] \& Oficina

Europea de Estadística

[Eurostat].

2018 Oslo Manual 2018: Guidelines for

Collecting, Reporting and Using Data on

Innovation. Cuarta edición. Luxemburgo:

OECD Publishing.

\section{Riikkinen, M., Saraniemi, S., \& Still, K.}

2019 FinTech as Service Innovators -

Understanding the Service Innovation

Stack. International Journal of E-Business Research, 15(1), 20-37. doi: 10.4018/

ijebr.2019010102

\section{Rojas, L.}

$2016 \quad N^{\circ} 24$. La revolución de las empresas

FinTech y el futuro de la Banca. Disrupción tecnológica en el sector financiero.

Caracas: CAF - Banco de Desarrollo de América Latina.

\section{Rojas, L.}

N²5. Transformación digital e innovación abierta en la banca. El caso del BBV.

Disrupción tecnológica en el sector financiero. Caracas: CAF - Banco de Desarrollo de América Latina.

\section{Rosenberg, N.}

1982

Inside the Black Box: Technology and

Economics. Cambridge: Cambridge

University Press.

\section{Saunders, M., Lewis, P., \&}

\section{Thornhill, A.}

Research methods for business students.

Quinta edición. Essex: Pearson.

\section{Seclen Luna, J. P.}

2016

Gestión de la innovación empresarial: un enfoque multinivel. 360: Revista de Ciencias de la Gestión, 1(1), 16-36.

\section{Seclen Luna, J. P.}

2019 Relationship between innovation process and innovation results: An exploratory analysis of innovative Peruvian firms. En J. Gil-Lafuente, D. Marino y C. Morabito (eds.), Economy, Business and Uncertainty: New ideas for a EuroMediterranean Industrial Policy (pp. 158171). Cham, Suiza: Springer Nature.

\section{Seclen Luna, J. P., \& Ponce Regalado, $\mathrm{F}$.}

Caracterización del proceso de innovación de empresas innovadoras peruanas: un análisis exploratorio y descriptivo. En C. Chávez-Rodríguez y C. Garrido-Noguera (coords.), La vinculación universidadempresa para el desarrollo integral con impacto social (pp. 436-453). Ciudad de México: REDUE-ALCUE, UDUAL.

\section{Seclen Luna, J. P., \& Barrutia-} Güenaga, J.

Gestión de la innovación empresarial: conceptos, modelos y sistemas. Lima: Fondo Editorial PUCP (en prensa). 


\section{bibliografía}

Shim, Y., \& Shin, D.H.

2017

Analyzing China's Fintech Industry from

the perspective of actor-network theory

Telecommunications Policy, 40(2/3), 168-

181.

\section{Sundbo, J., \& Gallouj, F.}

1998

Innovation in services. The results of Work

Package 3-4 of the SI4S project. Julio.

Recuperado de: https://www.researchgate. net/publication/278630108_Innovation_in_ services_in_seven_European_countries_ the_results_of_work_packages_3-4_of_ the_SI4S_project

\section{Sutherland, J.}

2010

Jeff Sutherland's Scrum Handbook.

Boston: Scrum Training Institute.

\section{Tether, B.}

Services, Innovation, and Managing

Service Innovation. En M. Dodgson, D.

Gann y N. Phillips (eds.), The Oxford

Handbook of Innovation Management.

Nueva York: Oxford University Press. doi:

10.1093/oxfordhb/9780199694945.013.031

\section{Thompson, B. S.}

Can financial technology innovate benefit distribution in payments for ecosystem services and REDD? Ecological Economics, 139(1), 150-157.

Tidd, J., Bessant, J., \& Pavitt, K.

Managing Innovation: Integrating

Technological, Market and Organizational

Change. Nueva York: John Wiley \& Sons.
2003

Yin, R

Case Study Research: Design and Methods. Thousand Oaks, California: Sage Publications.

\section{Varga, D.}

2017

FinTech, the New Era of Financial Services. Vezetéstudomány / Budapest Management Review, 48(11), 22-32.

\section{Wonglimpiyarat, J.}

FinTech banking industry: A systemic approach. Foresight, 19(6), 590-603.

Fecha de recepción: 25 de agosto de 2019 Fecha de aceptación: 10 de setiembre de 2019

\section{Tidd, J., \& Bessant, J.}

2009 Managing Innovation: Integrating

Technological, Market, and Organizational

Change. Cuarta edición. Chichester, 